\title{
Peter Handke: Literatur und Ethik
}

Tanja Angela Kunz: Sehnsucht nach dem Guten. Zum Verhältnis von Literatur und Ethik im epischen Werk Peter Handkes. Paderborn: Wilhelm Fink Verlag 2017 (=Ethik - Text - Kultur 12), 487 S.

Der Literatur-Nobelpreis für das Jahr 2019 hat die Kontroverse um den Schriftsteller Peter Handke noch einmal aktualisiert: diesmal im Rampenlicht der hohen kulturpolitischen Weihe. Ein zentraler Streitpunkt der aufgewärmten Debatte ist der angemessene Umgang mit Handkes 'projugoslawischem ` Engagement; ist für dieses doch charakteristisch, dass es die gängige Unterscheidung von literarischem und faktualem Diskurs, von ästhetischem und politischem Handeln auf eine besonders produktive oder eben besonders irritierende -, auf jeden Fall aber provokative Weise in Frage stellt.

Reduziert man die tatsächlich in ungewöhnlichem Maß polarisierte Debatte auf einen simplen Gegensatz, so zeichnet sich die folgende polemische Konfrontation ab. Auf der `Handke-kritischen $\$ Seite dominiert eine referenz- und kontextbezogene Perspektive: Handkes Jugoslawien-Komplex wird eine skandalöse politische Botschaft entnommen, die in letzter Konsequenz, und zwar in allen literarischen und nicht-literarischen Formaten des Engagements auf je eigene, doch semantisch korrespondierende Weise, auf einen wirren und menschenverachtenden Revisionismus bezüglich der jugoslawischen Zerfallsgeschichte hinausläuft. Auf der >Handke-affinen Seite dominiert hingegen eine `textimmanente Perspektive: Es wird auf der besonderen und einmaligen, den Gesamtkomplex prägenden ästhetischen und medienkritischen Motivation insistiert und folgerichtig eine Konzentration auf deren eigentlichen Wirkungsbereich betrieben: die eminent literarischen (essayistischen, epischen und dramatischen) Texte, die von den nicht-literarischen Phänomenen (Aussagen und Taten der Privatperson P. H.) in analytischer Sicht zu unterscheiden seien. 
Die Kontroverse drängt zu der allgemeinen Frage nach dem Verhältnis von ästhetischer Autonomie und gesellschaftlicher Verantwortung, und im Besonderen zu der Frage nach der ethischen Dimension von Handkes Werk und Wirkung. Unter welchen Umständen wird einem literarischen Verfahren, wird einem Text, wird einem Autor, wird einem öffentlichen Auftritt künstlerische Autonomie attestiert, unter welchen Umständen hingegen moralische Qualität bzw. öffentliche Verantwortung abverlangt? Unter welchen Bedingungen ist der Autor für die Wirkung seiner Texte, seiner Aussagen, seiner Aktionen unmittelbar verantwortlich? Wie steht es um die ethischen Intentionen in Handkes Opus, wie verhalten sich diese zu der in den Texten angelegten Wirkung, und wie schließlich zu der tatsächlich erfolgten Rezeption? Eine fundierte Diskussion dieser Fragen ist auf literatur- und kulturwissenschaftliches Expertenwissen $(\mathrm{m} / \mathrm{w})$ angewiesen.

Der Monographie von Tanja Angela Kunz kommt in diesem Zusammenhang der Status einer grundlegenden Forschungsarbeit zu, die sich von früheren Beiträgen zur vorliegenden Problematik durch eine übergreifende Perspektive und eine Anknüpfung an die Kontroverse abhebt: Es geht um eine Gesamtinterpretation von Handkes (vor allem, aber nicht nur) epischem Werk unter dem Gesichtspunkt der ethischen Dimension seiner Poetologie. Im Folgenden sollen Anlage und Ergebnisse der Monographie in ihren Grundzügen vorgestellt und abschließend diskutiert werden, welche Fragen damit abgedeckt, welche hingegen ausgeklammert oder aber provoziert werden.

Die Untersuchung versteht sich als Beitrag zu einer allgemeinen Fragestellung - »Inwiefern darf, soll oder kann ein künstlerisches Werk ethische Relevanz erlangen? « -, die am konkreten Opus exemplarisch abgehandelt wird: nämlich »vor dem Hintergrund eines moralisch strittigen[,] zugleich aber durch seinen modellhaften Anspruch in höchstem Maße ethisch aufgeladenen Werks« (S. 13). Die Diskrepanz von Anspruch und Rezeption in Handkes Fall lässt nun, so Kunz, das Unterfangen als problematisch erscheinen. Denn zum einen wird mit der moralischen Diskreditierung auch die bis dahin dominierende Sicht des Werkzusammenhangs in Frage gestellt. Zum anderen erscheint gerade der hier anvisierte, alternative, »tiefer in der Struktur der Werke«, also `unterhalb der Ebene einzelner Werkaspekte oder Handlungsmotive angelegte Kohärenzfaktor - nämlich der »Nachweis eines ethischen Schreibprojekts « - angesichts der »Debatte um die Unmoral der Jugoslawien-Schriften « gleichsam `verunmöglicht « zu sein (S. 16). Also muss die vorliegende Untersuchung zum einen die »ethische Integrität « auch der Jugoslawien-Schriften nachweisen, zum anderen »statt eines Bruchs die Konstanzen zu den übrigen Werken« aufzeigen (ebd.). 
Der Umsetzung des ambitionierten Vorhabens ist eine überzeugende Gesamtanlange, beachtliche Kenntnisse der Forschungslage und eine breit gefächerte theoretische Fundierung zu attestieren; nicht zuletzt auch eine erfreuliche Verbindung von Akribie und argumentativer wie auch stilistischer Transparenz. Im ersten Kapitel (»Einleitung«) werden die Grundzüge einer Forschungsgeschichte (nach 1945) zum Verhältnis von Literatur und Ethik im Bereich von Theologie, Philosophie und Literatur-/Kulturwissenschaft skizziert, die auf eine zunehmende Intensivierung im Zeichen eines >ethical turn « und einer `narrativen Ethik« hinausläuft. Im Anschluss werden »[z]eitgenössische Perspektiven auf das Verhältnis von Ethik und Ästhetik « (S. 22) im Spannungsfeld von Philosophie und Literaturwissenschaft diskutiert. Die Untersuchung zu Handkes Opus wird hier und in der Folge stringent ins Verhältnis zu fokussierten Aspekten der theoretischen Diskussion gesetzt. Der »intendierten Offenheit moderner Moralkonzepte « soll dabei eine Offenheit in der "Analyse des Beziehungsgeflechts von Literatur und Ethik« bei Handke entsprechen (S. 33): Die Untersuchung kann nicht an universellen moralischen Prinzipien ausgerichtet werden, sondern gilt dem Verhältnis von Ethik und Ästhetik in Handkes Werk, »in dem explizit oder implizit ethische Reflexionen vorgenommen, Wege zur Selbst-Verbesserung und zur Gemeinschaft mit Anderen gesucht werden sowie eine gezielte Leitung des Leserblicks zum Zweck der Erzeugung von Bewusstheit erfolgt« (ebd.).

Im zweiten Kapitel (»Grundlagen zu Historie und Werk«) werden die Grundkonzepte der Untersuchung, aufbauend auf Begriffs- und Forschungsgeschichte, sondiert und vorgestellt: die Leitbegriffe `Sehnsucht` (in Bezug auf Handke verstanden als ein positiv konnotiertes Grundgefühl mit katalytischer Funktion für ein ethisches Schreiben) und `das Gute` (nicht als Absolutheitskategorie genommen, sondern durch Merkmale der Fragmentierung, der Subjektbezogenheit und der Verbindung mit intellektueller Verantwortung bestimmt). Im gleichen Kapitel wird die etablierte Periodisierung von Handkes Gesamtwerk einer kritischen Revision unterzogen, als Voraussetzung für die hier ausgearbeitete Alternative eines auf thematischen Analogien im Bereich der Sehnsucht nach dem Guten beruhenden Werkzusammenhangs. Gesondert nachgegangen wird zwei von der bisherigen Forschung behaupteten Werk-Zäsuren, die mit einem Bruch in der Rezeption einhergingen: den Variationen des »Narzissmus-Vorwurfs«ab Ende der 1970er-Jahre (S. 47ff.) und der »Wende zu autorzentrierten und autobiographischen Studien« im Zuge der »Jugoslawien-Debatte« (S. 54ff.). Anschließend werden die Rezeptionsbrüche in einen weiteren Kontext gestellt, nämlich jenen des gesellschaftlichen und kulturellen Wandels zwi- 
schen »Utopismus und Postutopismus« (S. 65) seit den 1970er-Jahren und verstärkt nach 1989. Dieser Wandel, so die These, ließ Handkes utopische Ausrichtung auch unabhängig von der werkgeschichtlichen Entwicklung zunehmend unzeitgemäß erscheinen.

Die folgenden zwei Hauptkapitel behandeln in zwei Durchgängen das Verhältnis von Literatur und Ethik in Handkes CEuvre: zunächst aus einer eher thematisch-werkgeschichtlichen, sodann aus einer eher systematischen Perspektive, die sich aus der Zusammenführung von Handkes Werk und zeitgenössischen Ethiken ergibt. Methodisch geht es in den Hauptkapiteln um ein >Close reading` der mal einzeln, mal kumulativ herangezogenen Primärtexte, kombiniert mit theoretischen Überlegungen etwa zum UtopieBegriff, zur Epiphanie oder zur Grenze. Ein besonderer Schwerpunkt liegt auf dem Wertbegriff bzw. auf Überlegungen zu ethischen und ästhetischen Werturteilen im Rahmen der analytischen Philosophie, die zum Aufbau interpretativer Analogien in Handkes Werk herangezogen werden.

Das dritte Kapitel (»Ausrichtung auf das Gute«) ist zunächst dem Nachweis charakteristischer »Repräsentationsformen des Guten « gewidmet, die nach dem »Prinzip der Wiederaufnahme« (S. 119) das Gesamtwerk in allen seinen Entwicklungsphasen prägen. Dies gelte somit auch für »zwei scheinbare Reibungspunkte« (S. 120): das gesellschaftskritisch-sprachspielerische Frühwerk und die moralisch diskreditierten Jugoslawien-Schriften. Der genannten Repräsentationsformen sind vier: 1. »Peripetien zum Guten « meinen das "unverhoffte Auftreten des Guten oder eines motivierenden Aufrufs zum Guten am Ende eines Textes vor der Folie des Negativen « (S. 126f.). 2. »Das Gute im Off« meint »textinterne An- oder Vorausdeutungen bzw. Verweise auf eine spätere Wiederaufnahme einer Thematik [...], die im Text selbst nicht zur Entfaltung bzw. zur Realisierung kommen « (S. 139f.). 3. "Das Gute als das Diaphane« (S. 150) zielt auf Formen der Wortlosigkeit, denen in Handkes »Ästhetik der Abwesenheit» eine "produktive Bedeutung « zukommt (S. 160). 4. In den topographisch gemeinten und somit an die Bewegung der Helden gebundenen "Zwischenräumen " schließlich hat das Gute seinen »eigentlichen Ort« (S. 169). Die Thematik des Guten als einer offenbar in der Abwesenheit, in der Wortlosigkeit und im Dazwischen wirksamen Erfahrung wird anschließend in Beziehung gebracht zu den Konzepten der Utopie, des Defizitären sowie der Grenzund Schwellenphänomene.

Das vierte Kapitel (»Ethische Schreibweisen«) ist »vier wesentlichen ethischen Problemfeldern» gewidmet, »die zum Gegenstand ethischer Reflexion in Handkes CEuvre werden « (S. 34). Der "grundlegende Untersuchungsparameter « (ebd.) ist (1) die »Ethik der Ästhetik» (S. 279ff.), 
wobei Einsichten zum Verhältnis ethischer und ästhetischer Werturteile bei F. Nietzsche, K. H. Bohrer und vor allem bei C. McGinn die Deutungsgrundlage ist, das Ziel aber eine Untersuchung des - so oft und so kontrovers diskutierten - entsprechenden Zusammenhangs bei Handke: Aus der ethischen Potenz des literarischen Stils ergibt sich die besondere »Bedeutung der Wahrnehmung, des Sehens und des künstlerischen Blicks sowie die damit verwobene sprachliche Umsetzung als Verwirklichung" (S. 298). Eine Spezifizierung der Problematik liegt bei der (2)»Ethik der Narration« vor (S. 305ff.). Zunächst werden, unter Rückgriff auf theoretische Entwürfe von M. C. Nussbaum und P. Ricœur, »vier Erfahrungsweisen im Zusammenhang mit dem künstlerischen Schaffen « unterschieden und an Handke exemplifiziert, angefangen vom Erfahrungsaustausch auf der Ebene der Produktion (bei dem Handkes `Gutheißungsgesetz` im Mittelpunkt steht), über den Austausch zwischen Text und Leser sowie der Erfahrung des Rezipienten mit sich selbst, bis hin zur »Reflexion des Schreibvorgangs auf der Textebene « - »ein Spezifikum der Werke Handkes« (S. 311). Anschließend wird, anhand dreier theoretischer Zugänge zum Verhältnis von serzählten Geschichten` und `Leben` (W. Schapp, A. Cavarero, J. Butler), nach dem Stellenwert der (Lebens-)Geschichten in Handkes Werken gefragt. Dabei wird auf vier Aspekte eingegangen: das Fehlen von Charakteren und Lebensgeschichten im klassischen Sinn, die Darstellung von `Lebens-StückGeschichten «, der Entwurf von >Modell-Ethiken « und die Rückkehr der Geschichte als $>$ Verstrickung (von Protagonist, Erzähler und/oder Rezipient).

Mit der »Ethik des Selbst« (S. 348ff.) und der »Ethik des Anderen« (S. 392ff.) knüpft die Untersuchung an Bezugsgrößen an, die sich aus den zeitgenössischen Ethiken ergeben. »Im Vordergrund neuzeitlicher Ethiken " steht nämlich der »Bezug zu und Umgang mit anderen Lebewesen «, der freilich »unmittelbar zurück auf das Individuum « verweist (S. 34f.). (3) Die Untersuchung der ethischen Relevanz des Selbstbezugs in Handkes Werken führt zu dem Fazit, dass »die Arbeit am Guten« hier »auf die Stimmigkeit eines Lebens mittels Selbstverbesserung gerichtet ist «, die nur "narrativ erfolgen « kann (S. 357). Dies wird anhand der folgenden thematischen Komplexe näher ausgeführt: Religiosität bzw. Spiritualität, Sehnsucht als ethisches Prinzip, Identität als >innere Transzendenz` und schließlich `ethischer Narzissmus $<$. Letzteres meint einen narrativen Selbstbezug, der »aus der dilemmatischen Selbstbefangenheit heraus[führt], indem [er] in einer Multiplizierung der Perspektiven auf das Ich für sich selbst und Andere zur Anschauung kommt (S. 391) - womit der gegen Handke erhobene Narzissmus-Vorwurf widerlegt erscheint. (4) Inwiefern »der Andere als ethische Qualifizierungsgröße» (S. 35) in Handkes Werken eine Rolle spielt, wird in 
der Analyse der folgenden Phänomene geprüft (jeweils an einem anderen Textbeispiel): Fremdheit des Anderen, Anschauungsästhetik, narrative Zeugenschaft und das Prinzip der poetischen Gerechtigkeit (dies am Beispiel der Jugoslawien-Schriften). Die Begriffe `Sehnsucht « und `Begehren werden im Zusammenhang mit der Philosophie von E. Levinas diskutiert, um abschließend die »Beschaffenheit der Sehnsucht nach dem Anderen in Handkes Werken insgesamt « (S. 443) zu charakterisieren.

Insgesamt ist der Verfasserin eine überzeugende, über die bisherige Handke-Forschung zum Thema hinausführende Interpretation des sethischen Schreibprojekts gelungen. Das poetologische Fundament von Handkes ästhetischem Fundamentalismus $\iota^{1}$ wäre demnach ein ethisches, genauer: Es ergibt sich aus der besonderen Verbindung von Ethik und Ästhetik.

Wie steht es nun um die Überzeugungskraft und die Reichweite der vorliegenden Studie angesichts der »Debatte um die Unmoral der Jugoslawien-Schriften «(S. 16)? Sowohl der Nachweis einer »ethische[n] Integrität« dieser explizit-intentional `friedensstiftenden`, eine eigene >poetische Gerechtigkeit zelebrierenden Schriften, wie auch der Nachweis der »Konstanzen zu den übrigen Werken« (ebd.) ist überzeugend erbracht worden, womit ein zentraler Anspruch der Studie erfüllt ist. Außerdem kann die intentionale Ethik der Jugoslawien-Schriften auf einer anderen analytischen Ebene betrachtet werden als die »Unmoral « dieser Texte; der Vorwurf der Unmoral mindert nicht die Überzeugungskraft der auf die intentionale Ethik ausgerichteten Interpretation.

Anders steht es allerdings um die Reichweite der Untersuchung: Die Diskrepanz zwischen der >friedensstiftenden< Intention und dem Rezeptionseffekt der >Unmoral öffnet auch ethisch relevante Fragen, deren Berücksichtigung in einer Untersuchung zum »Verhältnis von Literatur und Ethik« wenn nicht erwartet werden muss, so doch erhofft werden kann. Während die Untersuchung, im Kontext von Werkentwicklung und Wirkungsgeschichte, durchaus auf das Gesamtspektrum von Handkes Jugoslawien-Engagement eingeht (vgl. S. 54-65), wird die Analyse des rethischen Schreibprojekts` zum einen nur »auf die vom Autor [schriftlich] konzipierten Texte« (S. 36) und zum anderen - auch im Fall der Jugoslawien-Schriften ${ }^{2}$ - auf ihren literarischen Charakter beschränkt. ${ }^{3}$ Diese

1 Stefan Breuer: Ästhetischer Fundamentalismus. Stefan George und der deutsche Antimodernismus. Darmstadt: Primus Verlag 1996.

2 Berücksichtigt werden vor allem: Winterliche Reise zu den Flüssen Donau, Save, Morawa und Drina (1996), Sommerlicher Nachtrag zu einer winterlichen Reise (1996) und Unter Tränen fragend (2000).

3 Zur Begründung s. S. 62-65 und 125f. 
Grenzziehung ist arbeitsökonomisch sinnvoll, doch kann ihre Begründung nicht recht überzeugen. Die Autorin lässt sich einerseits in die Debatte um den aktualitätsbezogenen Aussagegehalt von Handkes Texten ein (»kein Anzweifeln von konkreten Einzelschicksalen und Erfahrungen von Gewalt und Gräueln«, S. 63), womit jene diskursive Grenze überschritten wird, die in der vorliegenden Untersuchung sonst streng gewahrt werden soll. Im Anschluss werden literarische Intention und Werkkohärenz als Argumente gegen die außerliterarischen Vorwürfe ins Feld geführt; damit fällt die Autorin hinter die Ergebnisse jener Forschungen zurück, die in textnahen Interpretationen die analytische Unhaltbarkeit einer exklusiv literarischen Einhegung von Handkes Jugoslawien-Schriften nachweisen. ${ }^{4}$

Die Beschränkung auf die literarische Dimension von Handkes Jugoslawien-Engagement ist vor allem deswegen zu beklagen, weil damit die allgemeine Frage nach der »ethische[n] Relevanz«, die ein künstlerisches Werk erlangen »darf, soll oder kann«, nicht geöffnet wird für jene Aspekte, die dieses »moralisch strittige[] « und zugleich »in höchstem Maße ethisch aufgeladene[] Werk[]« (S. 13) geradezu provoziert. Wird hier doch die Grenze von literarischem und politischem Diskurs auf eine Weise irritiert bzw. programmatisch negiert, die nicht nur zu interpretativen Verwerfungen, sondern auch zu der Frage nach der ethischen Relevanz genau dieser Irritation führen muss.

In ihrer Untersuchung, so die Autorin, ginge es nicht darum, »wie häufig von der Literaturkritik gefordert, die Literatur sin die Pflicht $\triangleleft$ zu nehmen oder zur Moralanstalt zurückzuführen« (S. 37). Dies ist in der Tat nicht die Aufgabe der Literaturwissenschaft. Wohl aber zählt zu ihren Desideraten eine umfassende, an der Grenze von literarischer und außerliterarischer Kommunikation nicht haltmachende Erklärung, wie es dazu kommen konnte, dass das Schaffen eines Autors seit mehr als 20 Jahren in einer einmaligen wirkungsästhetischen Volte stets `das Gute` will und stets >das Böse « schafft. Schließlich dürfte das »ethische Prinzip der intellektuellen Redlichkeit«, das »für Handkes Werke maßgebend ist« (S. 368), auch bei der programmatischen Unterwanderung von Diskursgrenzen erforschbar und untersuchungswürdig sein. Angemessene Perspektiven in dieser wissenschaftlichen Causa können freilich nicht die Tribunale der öffentlichen

4 Exemplarisch: Jürgen Brokoff: »Srebrenica - was für ein klangvolles Wort«. Zur Problematik der poetischen Sprache in Peter Handkes Texten zum Jugoslawien-Krieg. In: Kriegsdiskurse in Literatur und Medien nach 1989. Hgg.: C. Gansel, H. Kaulen. Göttingen: V\&R unipress 2011, S. 61-88; Boris Previšić: Literatur topographiert. Der Balkan und die postjugoslawischen Kriege im Fadenkreuz des Erzählens. Berlin: Kulturverlag Kadmos 2014, S. 241-327. 
Entrüstung liefern, wohl aber Textstilistik und Narratologie, Rezeptionstheorie, Transferforschung und Literatursoziologie, flankiert von seriöser Forschung zu den jugoslawischen Zerfallsprozessen. 